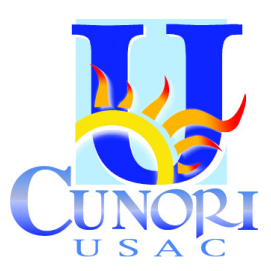

\author{
Revista Ciencia Multidisciplinaria CUNORI \\ http://revistacunori.com \\ DOI: https://doi.org/10.36314/cunori.v2i1.48 \\ ISSN: 2617- 474X (impresa) / 2617- 4758 (en línea)
}

Como citar el artículo

Arriola, C., Mazariegos, E., Arriola, C. \& Aceituno, M. (2018). Funcionalidad de la articulación acromioclavicular como resultado de reparación quirúrgica. Revista Ciencia Multidisciplinaria CUNORI, 2(1), 23-28. https://doi.org/10.36314/cunori.v2i1.48

\title{
Funcionalidad de la articulación acromioclavicular como resultado de reparación quirúrgica
}

\section{Functionality of the acromioclavicular joint as a result of surgical repair}

\author{
Carlos Arriola, Edvin Mazariegos, Carlos Arriola, Marvin Aceituno \\ Centro Universitario de Oriente -CUNORI, Universidad de San Carlos de Guatemala \\ Recibido: 01 de junio de 2018 Aceptado: 05 de agosto de 2018 \\ Disponible en internet el 17 de agosto de 2018
}

*Autor para correspondencia.

Correo electrónico: carlosarriola430@gmail.com

Resumen

$\mathrm{L}$ a luxación acromioclavicular grado $\mathrm{V}$ de Rockwood es una enfermedad ocasionada por traumatismo de alto impacto, produciendo dolor e incapacidad funcional. El tratamiento para este padecimiento es quirúrgico y se valora la calidad de vida de los pacientes con base en la funcionalidad del miembro superior afectado. La utilización de la escala de Western Ontario Shoulder Instability Index (W.O.S.I.) para evaluar la funcionalidad de la articulación acromioclavicular en pacientes que son tratados quirúrgicamente, el estudio permitió determinar, si el tratamiento implementado fue el adecuado para los pacientes y así mejorar la calidad de vida. Se realizó un estudio descriptivo transversal, donde se evaluó la funcionalidad del miembro superior de 30 pacientes con diagnóstico de luxación acromioclavicular grado $\mathrm{V}$ de Rockwood que fueron operados en el Hospital General de Accidentes “Ceibal". Se obtuvieron resultados por encima del $70 \%$, obteniendo un valor promedio de $86.76 \%$ de funcionalidad, resultado que demuestra una adecuada calidad de vida del paciente luego de ser sometido a una intervención quirúrgica. El tipo de técnica que más se utiliza en el Hospital General de Accidentes “Ceibal” fue la fijación coracoclavicular más suplementación acromioclavicular con un 63\% (n=19), siendo la segunda técnica la artroscopía, con un 37\% (n=11).

Palabras clave: western Ontario Shoulder Instability Index, funcionalidad, luxación, articulación acromioclavicular, calidad de vida

Abstract

Grade V acromioclavicular dislocation of Rockwood is a disease caused by high impact trauma, producing pain and functional disability. The treatment for this condition is surgical and the quality of life of these patients is assessed based on the functionality of the affected upper limb. The use of the Western Ontario Shoulder Instability Index scale (W.O.S.I.) to evaluate the functionality of the acromioclavicular joint in patients who are treated surgically allows us to determine if the treatment implemented was adequate for patients and thus improve their quality of life. A cross-sectional descriptive study was carried out, where the functionality of the upper limb of 30 patients diagnosed with grade V acromioclavicular dislocation of Rockwood who were operated in the General Accident Hospital "Ceibal" was evaluated. Results were obtained above 70\%, obtaining an average value of $86.76 \%$ of functionality, result that shows anadequate quality of life of the patient after being subjected to a surgical intervention. The type of surgical technique most commonly used in the General Accident Hospital "Ceibal" was the coracoclavicular fixation plus acromioclavicular supplementation with $63 \%(\mathrm{n}=19)$, with the second technique being arthroscopy, with $37 \%(\mathrm{n}=11)$. ). 
Keywords: western ontario shoulder instability index, functionality, dislocation, acromioclavicular joint, quality of life

\section{Introducción}

La luxación acromioclavicular tipo $\mathrm{V}$ en la clasificación de Rockwood se define como la ruptura de los ligamentos acromioclaviculares y coracoclaviculares con luxación de la articulación acromioclavicular y la clavícula extremadamente desplazada hacia arriba. (Ávila, 2015). El tratamiento de una luxación acromioclavicular depende de su clasificación según la escala de Rockwood, está clasificación consta de VI grados o tipos de luxación, considerando que el tratamiento para la luxación grado I y II son conservadores, las luxaciones que necesitan tratamiento quirúrgico son las IV, V y VI, dejando a las luxaciones de grado III en controversia en relación a su manejo, el cual puede ser conservador o quirúrgico (Cook, 2014).

La forma de evaluar un resultado postquirúrgico es evaluando la calidad de vida del paciente después de ser intervenido en sala de operaciones. Para poder determinar la calidad de vida de un paciente que es sometido a cirugía, es fundamental conocer que tan funcional es el miembro superior afectado y para ello esta investigación pretende usar la escala de Western Ontario Shoulder Instability Index (W.O.S.I.) para medir la funcionalidad del miembro superior que ha sido intervenido quirúrgicamente (Arcuri, 2012). Actualmente se disponen de varias herramientas para la evaluación de la calidad de vida después de los procedimientos quirúrgicos. En este caso se va a hacer hincapié en la escala de WOSI, la cual evalúa de manera específica la calidad de vida en los pacientes con antecedente de inestabilidad de hombro (Jaramillo, 2016).

\section{Material y métodos}

El estudio es de tipo descriptivo transversal en pacientes masculinos atendidos en la consulta externa del Hospital General de Accidentes "Ceibal" con diagnóstico de luxación acromioclavicular grado V de Rockwood sobre la funcionalidad de la articulación. Se evaluaron a 30 pacientes que fueron operados en el Hospital General de Accidentes "Ceibal" con dicho diagnóstico, a los cuales se les solicito completar el cuestionario W.O.S.I. Al tener los resultados, se determinó la funcionalidad del miembro superior según el puntaje obtenido en el cuestionario W.O.S.I. obteniendo resultados que determinaron la calidad de vida de los pacientes sometidos a intervenciones quirúrgicas.

También se determinó el tipo de técnica quirúrgica que fue utilizada con mayor frecuencia en el Hospital General de Accidentes "Ceibal" y que técnica provee mejores resultados funcionales para los pacientes. Así mismo se buscó identificar complicaciones postoperatorias que influyeran de manera negativa en la recuperación de los pacientes lo cual tendría repercusiones sobre la funcionalidad del miembro superior afectado.

\section{Resultados y discusión}

Se logró determinar la funcionalidad de la articulación acromioclavicular en 30 pacientes tratados quirúrgicamente por medio del puntaje obtenido en la escala de W.O.S.I. El puntaje es expresado en porcentajes, en donde el $100 \%$ se considera el nivel funcional máximo del hombro y $0 \%$ el nivel funcional 
mínimo. Demostrando que el 100\% (30) de los pacientes se encuentra por encima del 70\%, resultado que indica un alto nivel funcional. Se logró determinar que el puntaje máximo encontrado en el estudio fue de $99 \%$ y el puntaje mínimo fue de $72 \%$, ambos puntajes corresponden a resultados favorables para los pacientes.

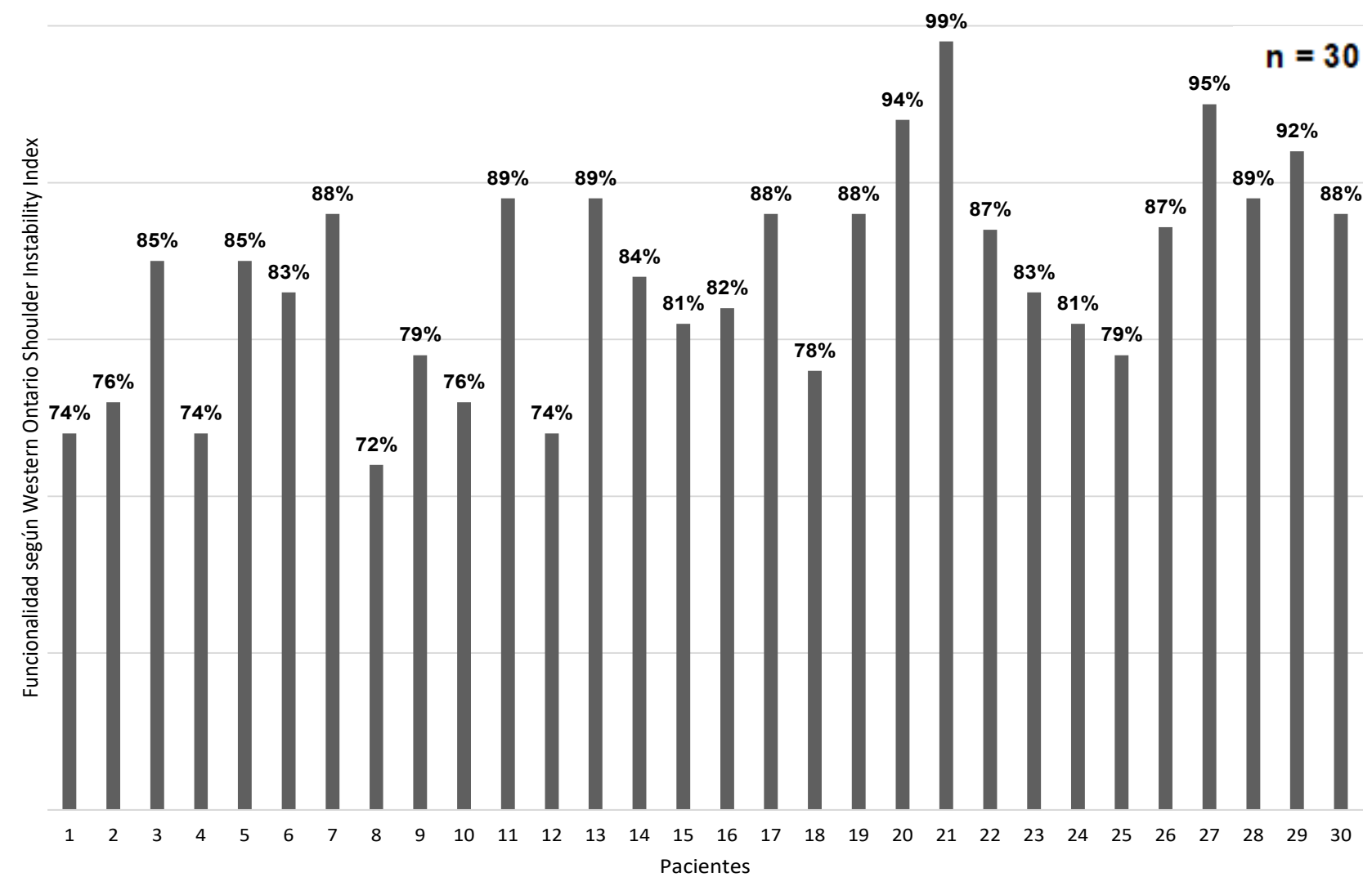

Figura 1. Funcionalidad del miembro superior afectado de los pacientes que fueron sometidos a intervenciones quirúrgicas con diagnóstico de luxación acromioclavicular gravo $\mathrm{V}$ de Rockwood en el Hospital General de Accidentes "Ceibal".

Un 63\% de los pacientes fueron intervenidos quirúrgicamente mediante la técnica de fijación coracoclavicular con anclaje, a esta técnica se le añade la suplementación acromioclavicular. Este procedimiento quirúrgico consiste en una serie de cuatro pasos fundamentales para la correcta reparación de la luxación acromioclavicular grado $\mathrm{V}$ de Rockwood, iniciando con la reducción de la luxación, seguida de la fijación coracoclavicular, en este punto se pueden utilizar distintos materiales o métodos, el más utilizado en el Hospital General de Accidentes "Ceibal" es el anclaje con FiberWire o sutura de ultra alto peso molecular. 
Al lograr la fijación coracoclavicular, sigue la plicatura muscular, que consiste en la reparación de la fascia del deltoides y trapecio, y para finalizar con el procedimiento quirúrgico se realiza protección con fijación acromioclavicular, para lo cual se puede utilizar un clavo Kirschner o Steinmann, según el criterio del cirujano. Únicamente el $37 \%$ de los pacientes evaluados en este estudio fueron intervenidos quirúrgicamente por artroscopia, siendo el método más utilizado el anclaje coracoclavicular con botón interno.

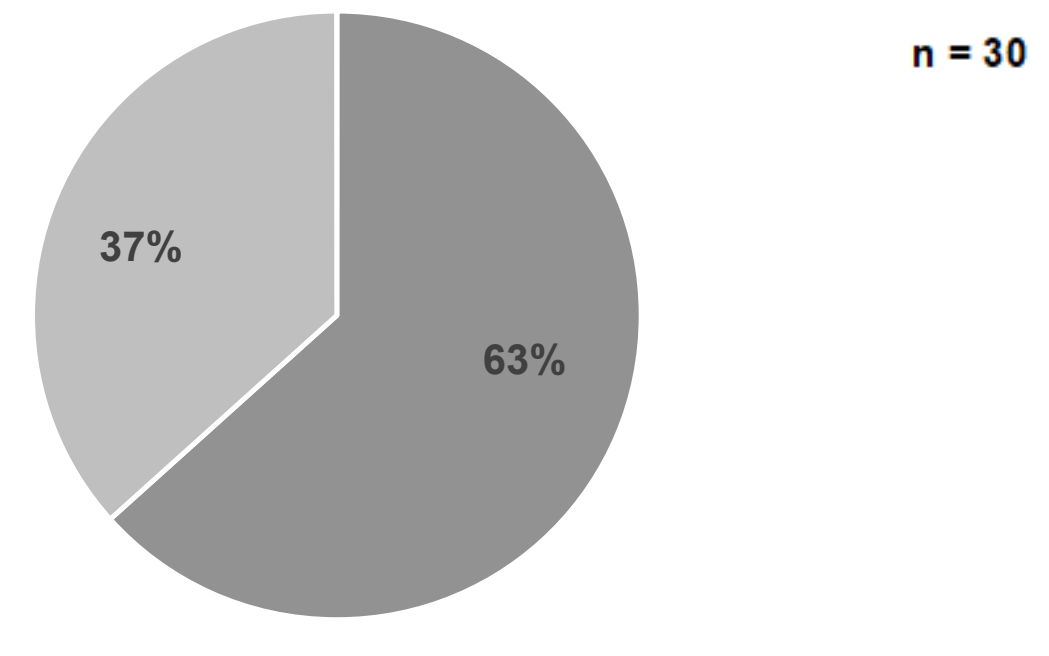

- Fijación coracoclavicular con anclaje $\quad$ Artroscopía

Figura 2. Tipo de técnica quirúrgica utilizada para el tratamiento de luxación acromioclavicular grado V de Rockwood en el Hospital General de Accidentes "Ceibal".

Se determinó que la técnica quirúrgica que provee mejores resultados para los pacientes fue la artroscopia, debido a que el resultado funcional se observa por arriba del $80 \%$, con un promedio de $88.54 \%$, lo que indica un nivel funcional óptimo. Sin embargo la fijación coracoclavicular más suplementación acromioclavicular resulta bastante efectiva, ya que los pacientes sometidos a este tipo de intervención tuvieron resultados por arriba del $70 \%$, con un promedio de $81.31 \%$. Pero a pesar de su alta eficacia, sigue siendo superior la operación por artroscopia, ya que demuestra mejores resultados en base a la funcionalidad del miembro superior afectado, lo que representa una mejor calidad de vida del paciente. 


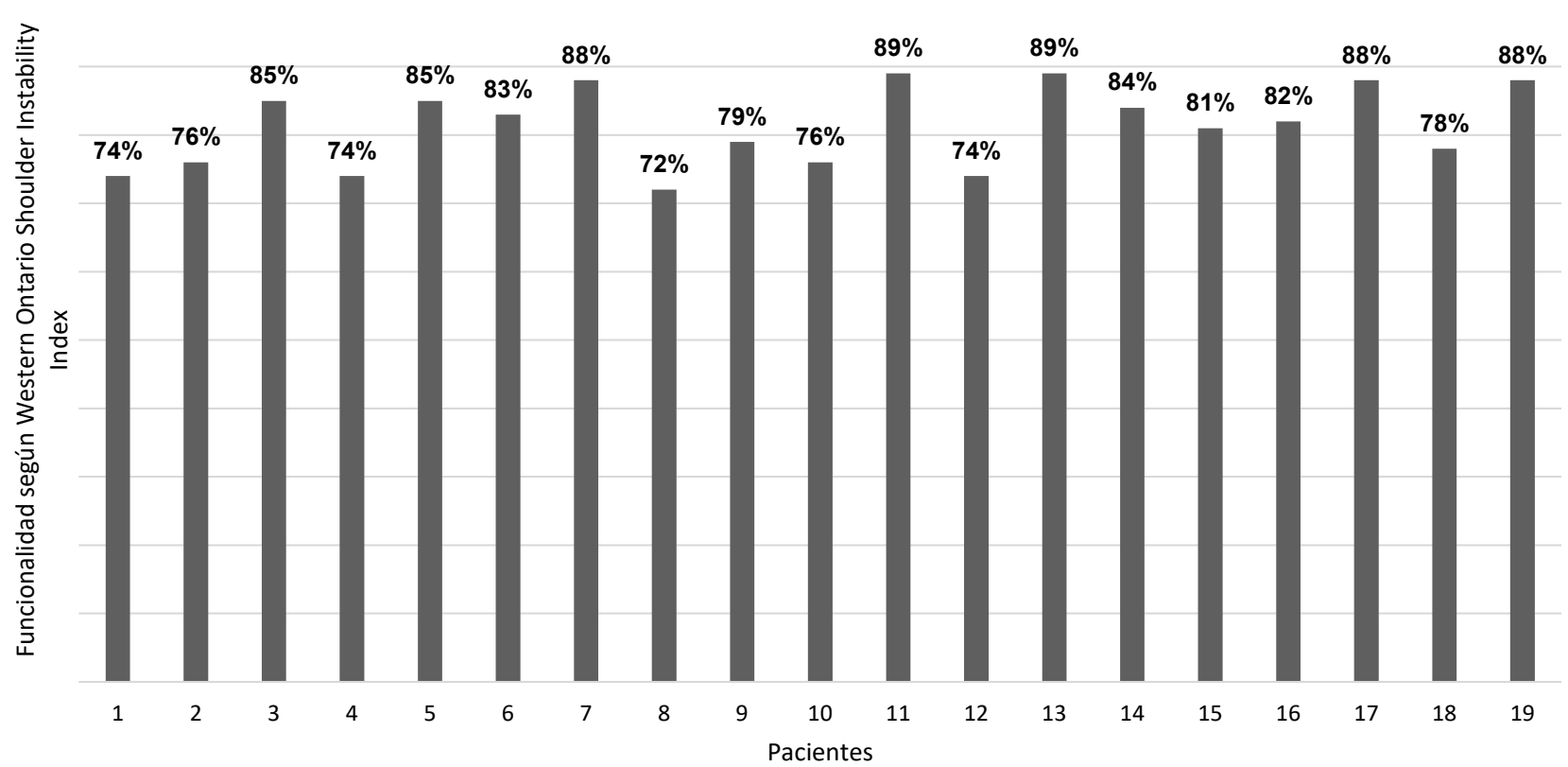

Figura 3. Funcionalidad de pacientes con luxación acromioclavicular grado $\mathrm{V}$ de Rockwood que fueron intervenidos quirúrgicamente por medio de la técnica de fijación coracoclavicular con anclaje en el Hospital General de Accidentes "Ceibal".

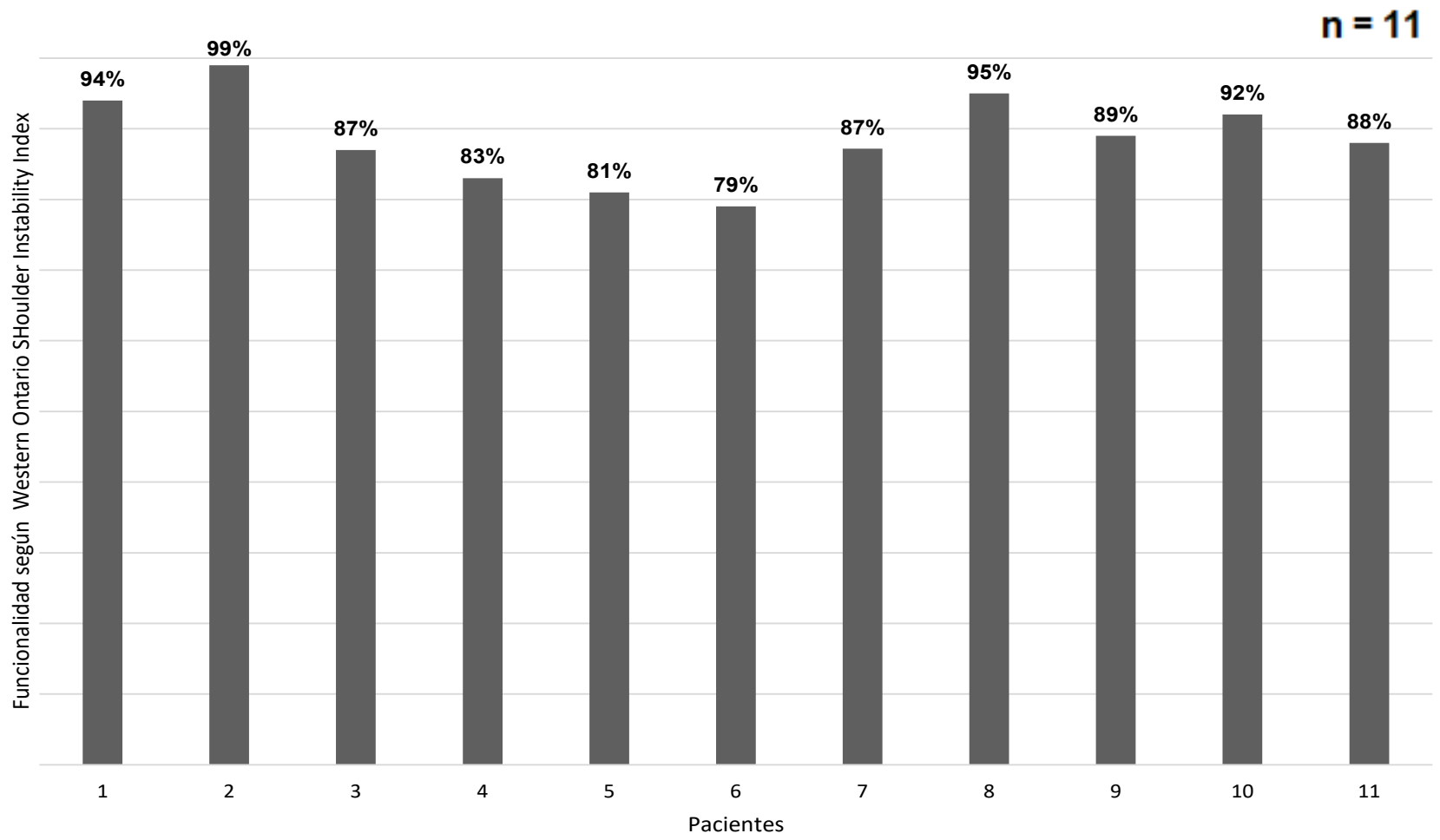

Figura 4. Funcionalidad de pacientes con luxación acromioclavicular grado $\mathrm{V}$ de Rockwood que fueron intervenidos quirúrgicamente por medio de la técnica de artroscopia en el Hospital General de Accidentes "Ceibal". 
Es importante mencionar que los pacientes dispuestos a ser evaluados eran los que presentaban un nivel de preocupación mayor por su patología o percibían algún déficit en comparación a su nivel previo a la luxación. También es necesario mencionar que los pacientes que tuvieron un mal puntaje en el test de W.O.S.I. tuvieron un mal cuidado dentro del primer año postoperatorio, como la participación en deportes de contacto y actividades de fuerza. A pesar de los déficits de rango observados en la población de estudio, los puntajes obtenidos en el test son altos, lo cual es indicativo del elevado nivel funcional que logran estos pacientes luego de la cirugía. Ninguno de los 30 pacientes en el estudio tuvo una complicación postoperatoria que limitara la funcionalidad de la articulación acromioclavicular.

\section{Agradecimientos}

A la Universidad de San Carlos de Guatemala y al Centro Universitario de Oriente por brindarme sus instalaciones para la formación como profesional, así mismo al Hospital de Accidentes "Ceibal” por el apoyo en la investigación.

\section{Referencias bibliográficas}

Arcuri, F., Abalo, E. \& Barclay, F. (2012). Uso de escores para evaluación de la inestabilidad de hombro. Revista de Artroscopia, 19(1), 67-72.

Ávila, VH., Pazmiño, J. \& Bravo, AX. (2015). Luxación acromioclavicular: tratamiento y complicaciones en el servicio de traumatología del Hospital José Carrasco Arteaga. Revista Médica HJCA, 7(3), 204-209. https://doi.org/10.14410/2015.7.3.ao.37

Cook, J. \& Tokish, J. (2014). Surgical management of acromioclavicular dislocations Clinics in Sports Medicine, 33, 721-737. https://doi.org/10.1016/j.csm.2014.06.009

Jaramillo F. \& Restrepo, C. (2016). Inestabilidad de hombro: una revisión de las opciones de manejo. Revista Colombiana de Ortopedia y Traumatología, 30(2), 55-60. https://doi.org/10.1016/j. rccot.2016.07.007

\section{Sobre el autor}

\section{Carlos Fernando Arriola Guerra}

Médico y Cirujano egresado del Centro Universitario de Oriente CUNORI de la Universisdad de San Carlos de Guatemala, realizó investigaciones sobre la "Funcionalidad de la articulación acromioclavicular como resultado de reparación quirúrgica”. Actualmente se desempeña trabajando en clínica privada en el municipio de Jocotán, Chiquimula.

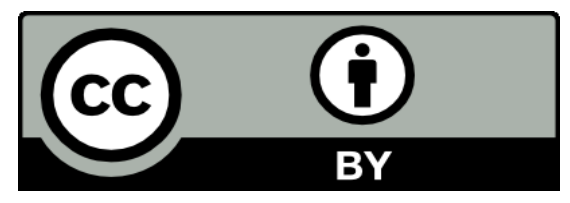

Este texto está protegido por una licencia CreativeCommons 4.0.

Usted es libre para compartir, copiar y redistribuir el material en cualquier medio o formato y adaptar el documento, remezclar, transformar y crear a partir del material para cualquier propósito, incluso comercialmente, siempre que cumpla la condición de atribución: usted debe reconocer el crédito de una obra de manera adecuada, proporcionar un enlace a la licencia, e indicar si se han realizado cambios. Puede hacerlo en cualquier forma razonable, pero no de forma tal que sugiera que tiene el apoyo del licenciante o lo recibe por el uso que hace. 